Europäische Akademie zur Erforschung von

Folgen wissenschaftlich-technischer Entwicklungen

Bad Neuenahr-Ahrweiler

Postfach 1460, D-53459 Bad Neuenahr-Ahrweiler

Tel.: + 49 (0) 2641/754300

Fax: + 49 (0) 2641/754320

》

\section{FGSV-Symposium "Kosten und Nutzen des Ver- kehrs - Neuere Entwicklungen der gesamtwirtschaftlichen Bewertung"}

\section{Tagungsbericht von Sigrid Klein-Vielhauer und Ekkehard Fulda, ITAS}

\section{Gesamtüberblick}

Die von der Forschungsgesellschaft für StraBen- und Verkehrswesen, Köln und Berlin, organisierte eineinhalbtägige Tagung, die am 17./18. Februar 1998 in Freiburg stattfand, fand bereits im Vorfeld großen Zuspruch - mehr als 200 Personen aus dem deutschsprachigen Raum hatten sich angemeldet. Auch bei den am Schluß des Symposiums plazierten Beiträgen und Diskussionen war noch ein äußerst großes Interesse der Teilnehmer zu konstatieren. Sicherlich ist dies im wesentlichen auf die Aktualität und Breite der angesprochenen und teilweise kontrovers diskutierten wissenschaftlichen Positionen zu gesamtwirtschaftlichen und gesamtgesellschaftlichen Bewertungsfragen in Verbindung mit der Errichtung und Nutzung verkehrlicher Einrichtungen zurückzuführen.

Der Tagung lagen verschiedene, sich zum Teil auch überschneidende Erwartungen zugrunde:

- Aktualisierung und Zusammenführung von verschiedenen Bewertungsverfahren im Hinblick auf die anstehende Fortschreibung des Bundesverkehrswegeplans für das Jahr 2001, vor allem des Verfahrens der Bundesverkehrswegeplanung, des Verfahrens für kleinräumig wirksame Straßenprojekte (früher RAS-W86 - Richtlinien für die Anlage von Straßen, Teil: Wirtschaftlichkeitsuntersuchungen, heute:
EWS - Entwurf für Empfehlungen für Wirtschaftlichkeitsuntersuchungen an Straßen, Ausgabe 1997), und des Standardisierten Bewertungsverfahrens, das bei Investitionen des öffentlichen Personennahverkehrs anzuwenden ist, die eine Gesamtkostenhöhe von mindestens 50 Mio. DM erreichen und für die Zuschüsse entsprechend dem Gemeindeverkehrsfinanzierungsgesetz beantragt werden;

- Suche nach einem gemeinsamen Korridor der quantifizierten Abschätzungen vor allem der verkehrsbedingten Umwelt- und Unfallwirkungen;

- Ermittlung der möglichen Bedeutung alternativer Bewertungsverfahren, die nicht primär auf die Monetarisierung der einzelnen Wirkungskategorien ausgerichtet sind.

Abgesehen von den einleitenden Vorträgen wurden je zwei oder drei Fachvorträge durch insgesamt fünf Podiumsdiskussionen unter Einbeziehung des allgemeinen Publikums ergänzt. Auf diese Weise vermittelte das Symposium einen anschaulichen Einblick in den derzeitigen Stand der verkehrswissenschaftlichen Bewertungsdiskussion im deutschsprachigen Raum.

Um das allgemeine Ergebnis gleich vorwegzunehmen: Einvernehmliche Antworten auf die Bewertungsfragen im Rahmen der Bundesverkehrswegeplanung und sonstiger Verkehrsplanungsaufgaben wurden - noch - nicht gefunden. Es bleibt abzuwarten, ob derzeit noch laufende Forschungsprojekte hieran Wesentliches ändern werden. Es scheint jedoch, daß die Vielfalt in den Vorgehensweisen beim Gesamtansatz sowie bei Einzelschritten eher zunehmen wird, und konkrete verkehrsbezogene Bewertungsaufgaben zumindest in den nächsten Jahren mit den bisherigen oder ad hoc modifizierten Methoden und Zahlenansätzen durchgeführt werden.

Um die Eigenheiten der derzeitigen verkehrswissenschaftlichen Bewertungsdiskussionen auch für mit der Thematik weniger Vertraute $\mathrm{zu}$ verdeutlichen, seien im folgenden einige Punkte herausgegriffen, die in den Vorträgen und Diskussionsbeiträgen des Symposiums mit unterschiedlichem Gewicht behandelt wurden, aber auch in der zukünftigen verkehrswissenschaftlichen Bewertungsdiskussion eine größere Bedeutung haben dürften. 
Die verkehrsbezogene Abwägungsaufgabe kann sich auf unterschiedliche Aufgabenstellungen beziehen, z.B. auf:

- Beurteilung von Einzelmaßnahmen

- Vergleich von Varianten

- Vergleich von Alternativen

- Dringlichkeitsreihung von Einzelmaßnahmen

- Beurteilung von Programmen/Plänen.

Eine formalisierte Kosten-Nutzen-Analyse sollte z.B. K. Beckmann, Aachen, zufolge auf die Dringlichkeitsreihung beschränkt werden. Häufig wird in der Verkehrswissenschaft im Hinblick auf die Bewertung zwischen der Erstellung der Infrastruktur und der Nutzung der Infrastruktur (=Verkehrsleistungen) unterschieden.

Im Mittelpunkt der Bewertungsdiskussion stehen die Kosten. Kostengrößen werden für unterschiedliche Zwecke gebraucht, z.B. als (Maibach, Zürich):

- Gesamtkosten, um Relationen zwischen verschiedenen Verkehrsträgern bzw. Verkehrsmitteln/Verkehrsmittelkombinationen sichtbar zu machen,

- Grenzkosten (= Kosten pro zusätzliche Einheit): zur Steuerung über den Preis, z.B. beim Kraftstoffverbrauch,

- Kostensätze, mit denen verschiedene Wirkungskategorien für formalisierte KostenNutzen-Analysen einheitlich in monetären Größen dargestellt werden.

Hinsichtlich der Kosten wird häufig auf die Notwendigkeit einer möglichst vollständigen Erfassung hingewiesen, die neben den internen vor allem auch die externen Kosten einschließt. Es gibt unterschiedliche Abgrenzungen von internen und externen Kosten: z.B. für W. Rothengatter, Karlsruhe, ist "extern" das, was sich außerhalb des Marktes ereignet, und für $\mathrm{H}$. Baum, Köln, das, was sich außerhalb des Verkehrssektors ereignet. Es gibt aber auch Tendenzen, nur politisch brisante Kostenaspekte zu berücksichtigen, bzw. Empfehlungen hierfür.

Der Unterscheidung von internen und externen Kosten kommt eine besonders große Bedeutung $\mathrm{zu}$, wenn in erster Linie die vollständig erfaßten externen Kosten als geeigneter Ansatzpunkt für die Steuerung über den Preis
(Internalisierung) gelten.

Darüber hinaus kommt bei derartigen $\mathrm{Be}$ trachtungen die mögliche Bedeutung von externen Nutzen ins Spiel, die einer Anlastung von externen Kosten entgegenstehen könnten. Als Nutzen verkehrlicher Aktivitäten wird z.B. von H. Baum vor allem die Sozialproduktsteigerung betrachtet. Hierbei wird abgebildet, was passiert wäre, wenn der Verkehr nicht so gewachsen wäre, wie er tatsächlich gewachsen ist.

Bei den auf die Beurteilung oder den Vergleich von Einzelmaßnahmen bezogenen Betrachtungen sind die Nutzenaspekte die Minderkosten, die gegenüber einem Vergleichsfall anfallen. Dies kann sich auf verschiedene Umwelt- und Unfallkosten, z.B. aber auch auf die Betriebskosten beziehen. In diesem Beispiel stellen die Investitionsausgaben die Kostenseite des Kalküls dar. Eine größere Bedeutung für das jeweilige Gesamtergebnis kann die Berïcksichtigung der eingesparten Fahrzeiten erlangen.

Für die Abbildung der externen Kosten bzw. der aus ihnen abgeleiteten Nutzenelemente in monetären Größen (Monetarisierung) werden üblicherweise drei Ansätze diskutiert:

- Reparaturkosten (=Ressourcenausfall-Ansatz)

- Schadensvermeidungskosten

- Zahlungsbereitschaft für Verbesserungen (=für nicht anfallende - externe - Kosten)

Trotz der schon früher geführten ausführlichen Diskussionen über die Vor- und Nachteile der verschiedenen Monetarisierungsansätze weichen die Einschätzungen, welcher Ansatz grundsätzlich zu bevorzugen ist, voneinander ab. Maibach hält darüber hinaus die Unsicherheiten bei der Monetarisierung für prinzipiell unüberwindbar und verweist darauf, daß verkehrspolitische Lenkung und Internalisierung nicht gegeneinander ausgespielt werden sollten.

Der in Kürze von der Forschungsgesellschaft für Straßen- und Verkehrswesen herausgegebene Tagungsband wird eine willkommene Gelegenheit sein, die hier angesprochenen und weitere Fragen zu vertiefen und insbesondere die Vor- und Nachteile nicht-monetarisierender Bewertungsverfahren für den Verkehrssektor weiter auszuloten. 


\section{Ausschnitte aus den Vorträgen und der Diskussion}

\section{Gesamtwirtschaftlicher Nutzen des Verkehrs}

Herbert Baum (Köln) stellte die "Nutzen" des Verkehrs heraus, die sich in gesamtwirtschaftlichem Wachstum niederschlagen, und zwar im Hinblick auf die wichtigsten Wachstumsfaktoren "Produktivität" und "Kapital": Der Verkehr mache rund $48 \%$ des gesamtwirtschaftlichen Wachstums in Deutschland seit 1950 aus, $26 \%$ habe allein der Straßenverkehr bewirkt. Zwei Drittel dieses Wachstums werde vom Güterverkehr bestritten, das restliche Drittel vom Personenverkehr. Ausgedrückt in absoluten Werten lasse sich 1989 ein gesamtwirtschaftlicher Wachstumsnutzen des Straßenverkehrs in Höhe von 404 Mrd. DM errechnen. Nach der InputOutput-Analyse mache etwa im Jahre 1996 der Beitrag des Güterverkehrs zum Volkseinkommen 123 Mrd. DM aus, der des Personenverkehrs $124 \mathrm{Mrd}$. DM, so daß sich insgesamt als Beitrag des Verkehrs zum Wirtschaftswachstum eine Größe von rund 250 Mrd. DM ergebe.

Werner Rothengatter (Karlsruhe) betrachtete in seinem Referat die Auswirkungen von Verkehrsinfrastrukturinvestitionen auf das regionale Wirtschaftswachstum. Erkennbar sei, daß eine standardisierte ökonomische Bewertung von Straßeninfrastrukturinvestitionen im Hinblick auf ihre regionalwirtschaftlichen Folgen bereits methodisch problematisch sei. Eine Bewertung von eventuellen Wachstumseffekten habe grundsätzlich auf der Netz- oder Systemebene zu erfolgen; es reiche nicht aus, nur Einzelprojekte zu bewerten. Eine pauschale Annahme, Straßenbaumaßnahmen seien effizient im Hinblick auf ökonomisches Wachstum, werde teilweise zwar auf der Makroebene gestiitzt. Ein differenziertes Bild ergebe sich jedoch bei regionaler Betrachtung. Verkehrsverändernde Maßnahmen könnten unter Umständen zu Nachteilen für die regionalwirtschaftliche Entwicklung führen, wenn durch die neuen Verkehrsinfrastrukturen die Agglomerationen noch verstärkt werden und die unterentwickelten Regionen vergleichsweise weiter in die Peripherie geraten. Demgegenüber gebe es sogar einen Spielraum für "Verkehrsverminderung": Über die Verminderung der Ballung kann sich eine Verbesserung der verkehrswirtschaftlichen Effizienz ergeben.

Über Arbeitsmarkt- und Beschäftigungseffekte "erwogener Maßnahmen" in der Verkehrsplanung referierte Zachcial (Bremen) aus Erhebungen in der Bauphase von Bundesautobahnabschnitten und -Kreuzen, Neubauabschnitten wie auch Ergängzungsbauten. Bezüglich Beschäftigungseffekten ergab sich insgesamt zwar ein differenziertes Bild, doch könne man jedenfalls resümieren: "Eine arbeitsplatzerhaltende Funktion des Autobahnbaus ist eindeutig da!"

In der Diskussion fragte Sandhäger (BMV, Bonn) nach einem "Korridor der Plausibilitäten" im Hinblick auf den Zusammenhang von Wirtschaftswachstum und Verkehrsentwicklung. Dabei ließ sich zwar als Konsens ausmachen, $d a \beta$ es Wirkungen des Verkehrs auf wirtschaftliches Wachstum gebe. Kontrovers wurde aber die Frage beantwortet, in welchem Maße Wirtschaftswachstum durch Verkehr gefördert würde.

Wolfgang Blum (Dresden) sprach von einem 5-10\%igen Einfluß, wies aber auf Alterungseffekte hin, die zu berücksichtigen seien wie auch auf Rückwirkungen, insbesondere indirekte Rückspuleffekte, die nur schwer einschätzbar blieben. Ihde (Mannheim) betonte nachdrücklich die Wachstumswirkungen des Verkehrs, gleichzeitig aber die Schwierigkeit, diese auch zu quantifizieren. Das Ausmaß der Wirkungen des Verkehrs auf wirtschaftliches Wachstum sei jedoch in der Regel viel größer, als es benannt würde. Seine pointierte These: "Wer Verkehr verhindert oder über seine Kosten hinaus verteuert, verhindert Wachstum oder verlagert Wachstum anderswohin."

Rothengatter betonte das Erfordernis einer differenzierten Betrachtung. Da das gesamte Verkehrsnetz in unserer Wirtschaft ja schon vorhanden sei, wäre eine Marginalitätsbetrachtung eher angemessen. Bezüglich einer Quantifizierung von Wachstumseffekten sei zu differenzieren: Zwar würden reine Transportkosten sinken, aber Logistikkosten insgesamt würden eher steigen. Nach Weltbank-Studien seien positive Effekte auf das Wachstum insbesondere erkennbar, wenn Engpässe vorhanden und Reduktionen der generalisierten Verkehrskosten groß seien. Die Untersuchungen hätten vor allem gezeigt, daß Verkehrsinvestitionen nicht 
als Einzelmaßnahmen betrachtet werden dürften, sondern daß die Wirtschaft auch noch mit anderen Maßnahmen zu fördern sei. Bei isolierten Verkehrsinfrastrukturmaßnahmen könnten sogar kontraproduktive Effekte auftreten.

\section{Schadstoffemissionen; Unfälle; externe Ko- sten und externe Nutzen}

Christoph Walther (Basel) erläuterte die neuen Bewertungskriterien der EWS '97 im Vergleich zur RAS-W '86, wobei er hervorhob, daß insbesondere Klimaschäden mitberücksichtigt werden durch die Erfassung von $\mathrm{CO}_{2}$-Emissionen; auch Immissionskonzentrationen einschließlich Schäden an der Vegetation finden dabei Berücksichtigung. Bezüglich der Emissionen von Luftschadstoffen wird in den EWS in $\mathrm{NO}_{x^{-}}$ Äquivalenten umgerechnet. Allgemein werde auch bei den EWS davon ausgegangen, daß der Vermeidungskostenansatz nicht wählbar gewesen sei, daß man vielmehr den Schadenstatbestandsansatz zu verwenden hatte.

Über die Angemessenheit der Bewertungsverfahren in den EWS für Schadstoff-Emissionen wurde vor allem im Hinblick auf Umweltaspekte heftig gestritten. Friederich (UBA/Berlin) nannte die EWS-Methoden von Schadstoff-Emissionen "sachlich falsch!" Vor allem sei $\mathrm{NO}_{\mathrm{x}}$ keine geeignete Leitkomponente, um die Schädlichkeit verschiedener Schadstoffe darzustellen. Außerdem sei es falsch zu sagen, man könne keine Vermeidungskosten ansetzen. Auch Udo Becker (Dresden) kritisierte die Bewertungen schädlicher Emissionswirkungen anhand von $\mathrm{NO}_{\mathrm{x}}$-Äquivalenten und meinte, das "sollte in der Weiterentwicklung der EWS gestrichen werden". $\mathrm{NO}_{\mathrm{x}}$ - Äquivalente als Bewertungsmaßstab wurden dagegen von Cervenka (Wien) verteidigt unter dem Hinweis, daß es derzeit dazu keine Alternative gebe, da konstruktive Verbesserungsvorschläge fehlen würden.

Zur Bewertung von Straßenverkehrsunfällen wurde von Wünsche (Köln) ein neues Berechnungsmodell vorgestellt, das viele Ergänzungen und zum Teil erhebliche Modifikationen gegenüber dem bisherigen aufweist. Es führt aber in den Größenordnungen zu ganz ähnlichen Ergebnissen wie die Berechnungen nach EWS. Nach dem alten wie nach dem neuen Berechnungsverfahren ergibt sich für die
Gesamtkosten aus Verkehrsunfällen eine Größenordnung von ca. 26 Mrd. DM. Nach Wünsches Auffassung wären für eine Weiterentwicklung der Berechnungsverfahren insbesondere die "humanitären Unfallfolgen" zu ergänzen, d.h. die durch Unfälle verursachten Außermarktkosten, z.B. psychische Beeinträchtigungen oder unfallbedingte Veränderungen der Lebensplanung, wenn eine Ausbildung nicht durchgeführt werden kann. Aus derartigen humanitären Kosten könne eine geringere Leistungsfähigkeit resultieren, was entsprechend in Geldeinheiten anzusetzen wäre. Nach seinen Schätzungen lassen sich für das Jahr 1994 beispielsweise 7,2 Mrd. DM an humanitären Kosten berechnen.

Kontrovers verlief die Diskussion von externen Kosten und von externen Nutzen. Maibach (Zürich) kam in seinem Referat über externe Kosten des Verkehrs in der Schweiz zu den Schlußfolgerungen: Externe Kosten ließen sich nicht wirklich im Detail berechnen, es könne sich vielmehr nur um grob überschlägige Verfahren handeln. Bezüglich des unter Umweltaspekten sensibleren Alpenraums ergäben sich spezielle Bewertungsprobleme. Insgesamt handele es sich bei dem Konzept externer Kosten um "stark anthropozentrische Ansätze", mit denen man gerade die Schäden der Biosphäre schlecht einschätzen könne. Der Umweltproblematik werde man letztlich mit einer Reduktion auf Geldeinheiten nicht gerecht. Aber auch angesichts dieser methodischen Schwächen wollte Maibach nicht ganz auf die Abschätzung externer Kosten des Verkehrs verzichten, denn der Internalisierungsansatz bilde einen wesentlichen ökonomischen Baustein für die Nachhaltigkeitsdiskussion.

Vor Einbeziehen von externen Kosten in die Bewertung von Verkehrsprojekten sei zu prüfen, meinte Friederich (Berlin), ob es eventuell Unersetzbarkeiten gebe, ob etwa Arten infolge von Verkehrsprojekten sterben würden; eine ausgestorbene Art könne man nicht mehr in pekuniäre Kosten-Nutzen-Abwägungen einrechnen. Seitens Dicke (VDA) wurde hervorgehoben, daß die Automobilindustrie bezüglich der Ressourcen einen effizienteren Umgang ermöglicht habe. Die externen Kosten hätten sich dramatisch zurückentwickelt insofern, als der Automobilverkehr heute ein Drittel weniger Abgase als noch 1991 produziere. Diese Ver- 
minderung sei ohne Abgabenpolitik auf ordnungsrechtlichem Weg erzielt worden. Dagegen forderte Friederich einen angemessenen Einsatz des Ordnungsrechts, weil nur dieses mit Aussicht auf einigen Erfolg angewendet werden könne, wenn es nachgewiesene externe Kosten des Verkehrs gibt.

Unversöhnlich standen sich die Standpunkte gegenüber angesichts der Frage, ob auch externe Nutzen des Verkehrs in die Internalisierungsberechnungen einzubeziehen wären. Für eine zu den externen Kosten analoge Behandlung von externen Nutzen plädierte W. H. Schulz (Köln), wobei seine Argumentation überwiegend im Rahmen allgemein gehaltener ökonomischer Zusammenhänge erfolgte. Paradebeispiel für externe Nutzen seien Ballungen von Industrieansiedlungen; Schulz sprach von einem "silicon-valley-Effekt". Die weitgehende Entkoppelung der Produktionsstätten von den Regionen der Nachfrage setze den Verkehr voraus. Durch die Zentrierung der Industrien entstünden verschärfte Konkurrenzen zwischen den Produktionsbetrieben etwa bezüglich ihrer Mitarbeitenden. Zwischen den Betrieben zirkuliere das technisch-organisatorische Wissen dann entsprechend schneller. Gegenüber einer weniger agglomerierten Industriestruktur entstünden damit für die Konsumenten externe Nutzen aus dem Verkehr in Form eines aufgrund der zugespitzten Konkurrenzsituation niedrigeren Güterpreises. Auch aufgrund neuer Technologien entstünden fortlaufend neue strategische Komplementaritäten, aus denen zunächst externe Nutzen resultierten, bevor sie jeweils internalisiert werden könnten. Daher seien externe Nutzen aus dem Verkehr auch analog zu dessen externen Kosten in die Bewertungen einzubeziehen. Diesem Fazit von Schulz mochte sich auf der Freiburger Tagung ein Großteil der Teilnehmenden nicht anschließen. Das Konzept externer Nutzen blieb umstritten.

\section{Monetäre Bewertungen von Verkehrs- projekten}

$\mathrm{Zu}$ den Grenzen und der Ergänzungsbedürftigkeit von monetären Bewertungen von Verkehrsprojekten hat Klaus Beckmann (Aachen) pointiert Stellung bezogen; er stellte die "gesellschaftliche Abwägung" als eine Alternative zur monetären Bewertung dar. Seine zentrale These: "Monetäre Bewertungen können gesellschaftliche Abwägungen nicht ersetzen, sondern 'nur' unterstützen." Die in den Bewertungsansätzen von BVWP und EWS vorgenommene Monetarisierung sei in mehrfacher Hinsicht voreilig; man solle im Entscheidungsprozeß länger bei Wirkungsbeschreibungen bleiben, um häufige Fehler bei Beurteilungen, Abwägungen und Auswahlen zu vermeiden. Sowohl im Hinblick auf die Weiterentwicklung der BVWP als auch derjenigen der EWS seien multikriterielle Abwägungsverfahren stärker zu diskutieren. Bewertungsverfahren hätten Möglichkeiten zu eröffnen insbesondere für: kognitive Behandlung, vor allem um Lernprozesse zu fördern; angemessene kommunikative Behandlung; verbal-argumentative und diskursive Behandlung in 'gewohnter' Formulierung; Behandlung mit 'gewohnten' Auswahlverfahren; sowie für einen beteiligungsorientierten Ansatz. Nach Beckmann lautet die wichtigste Schlußfolgerung: "Wir brauchen immer einen Methodenmix! - Die Kosten-Nutzen-Abwägung ist nur bei der Dringlichkeitsreihung von Projekten uneingeschränkt relevant." Beckman regte an, Maßnahmenbewertungen mit dem von ihm vorgeschlagenen Verfahren durchzuführen wie parallel auch mit monetarisierenden Verfahren in Anlehnung an BVWP und die EWS, um an konkreten Projekten die Bewertungsergebnisse dann ex post evaluieren zu können.

In der Diskussion über den Stellenwert der Monetarisierung kamen u.a. Rückkopplungsprozesse zur Sprache: Verkehrliche Maßnahmen haben Rückkopplungen zur Folge, insbesondere den "induzierten Verkehr"; derartige Folgeeffekte seien häufig nicht monetarisierbar. Die Verwendung eines Methodenmixes sei, so sagte Dobeschinsky (Stuttgart) nichts prinzipiell Neues, denn das Standardisierte Verfahren für ÖPNV-Projekte kenne ja die Vorgehensweise, daß eine Linie bestimmt werde, an der die reine Berechnung aufhöre und argumentative Erörterungen einzusetzen hätten. Der Diskussionsleiter, Meewes (Köln), nannte den Konsens "ein schönes Ergebnis", daß zunächst einmal die Wirkungsbeschreibung in originären Dimensionen zu erfolgen habe.

Moosmayer (BMV/ Bonn) führte in seinem Beitrag über "Ansätze für eine Weiterentwicklung des Bundesverkehrswegeplanes" aus, 
daß verschiedene Arten von Verkehrswegen zu vergleichen seien. Angesichts heterogener Dimensionen sei vor allem Vergleichbarkeit herzustellen - dazu würden ökonomische Maßeinheiten als "Dach der Vergleiche" gebraucht. Nur über eine 'eindimensionale' Homogenisierung könnten ganz unterschiedliche Größen aggregierbar gemacht werden. Monetarisierte Bewertungen dürften daher nicht vorschnell aufgegeben werden, wenn man noch zu politischen Entscheidungen kommen wolle. Moosmayer betonte, über "Wettbewerbspreise" könnten auch immaterielle Werte wie gerade solche der Umwelt gefaßt werden. Außerdem müßten ökonomische Effekte in Marktpreisen ausgedrückt werden.

\section{Aktualisierungen der Bewertungsverfahren; Aussichten auf ihre Zusammenführung}

Es herrschte Konsens darüber, daß zwischen den verschiedenen Bewertungsverfahren EWS, BVWP und Standardisierte Bewertung eine Harmonisierung vorzunehmen sei. Der methodische Stand der EWS 97 würde in die kommende BVWP auf jeden Fall eingearbeitet. Dies stelle noch nicht den endgültigen Ansatz des BVWP 2001 dar, werde vielmehr laufend weiterentwickelt. Wie Ulrich Brannolte (Weimar) darlegte, wird noch an der Lösung folgender Hauptaufgaben gearbeitet: Noch besser sei zu berücksichtigen, daß die Datenverfügbarkeit vom jeweiligen Planungsstadium abhängig ist. $\mathrm{Zu}$ verbessern sei auch, daß Planungen nicht synchronisierbar seien sowie, daß die Projekte in unterschiedlichen Reifegraden vorliegen. Im Laufe ihrer Konkretisierung verschlechtert sich das Nutzen-Kosten-Verhältnis in der Regel zunehmend; dies wäre bereits in die früheren Planungsstadien einzubeziehen. Das "Kostenmanagement" und die Nachbewertung der realisierten Projekte seien verbesserungsbedürftig. Gegenüber den EWS 97 seien noch keine anderen Verfahren erkennbar, die als grundsätzliche Alternative in Frage kommen würden.

Dobeschinsky forderte für eine Fortentwicklung der Standardisierten Bewertung (ÖPNV) vor allem, deren Einschränkung auf Verdichtungsräume zu überwinden. Auch Verkehrsprojekte im ländlichen Raum sollten einer entsprechenden rationalen Bewertung unterzogen werden. In vier Bereichen wären noch Er- gänzungen anzubringen: Fortschreibung der Kosten- und Wertansätze; Vereinfachung der Verfahren; Einführung von Verfahren für automatisierte Systeme; Übergangsverfahren für die Neuen Bundesländer. Wie Dobeschinsky betonte, würden die Verfahren zur Bewertung von ÖPNV-Projekten parallel zu denen der EWS weiterentwickelt, Fernziel sei aber, beide einmal zu einem einzigen Bewertungsverfahren zusammenzufügen.

Für die BVWP zeigte Moosmayer insbesondere die folgenden vier Punkte zur Verbesserung und Weiterentwicklung auf: Die intermodale Ausweitung habe auch in Hinblick auf See- und Luftverkehr zu erfolgen. Die TeleInformatik sei deutlich stärker auszubauen, vor allem sei die Frage zu klären, wie weit sie eingesetzt werden könne. Konjunkturneutrale Beschäftigungseffekte seien zu berücksichtigen und überhaupt die Wegebau-induzierten Förderprozesse für die wirtschaftliche Entwicklung in einer BVWP-Neufassung explizit abzubilden. Moosmayers Fazit: Ganze Bauprogramme sind zu bewerten, Bewertungen isolierter Einzelprojekte reichten nicht aus.

Bereits in seinem einleitenden Referat hatte Sandhäger (BMV/Bonn) darauf hingewiesen, daß die Hauptkriterien der Weiterentwicklung von Bewertungsmaßstäben in der BVWP freilich von der neuen Bundesregierung $a b$ Herbst 98 festgelegt würden. Die Wissenschaft könne einstweilen nur vorbereitend arbeiten. Wie auch immer im einzelnen die Einschätzung von Kosten und Nutzen des Verkehrs vorgenommen werden würde - das zentrale Ziel der Verkehrspolitik sei es, eine Entkoppelung von Wirtschaftswachstum und Verkehrswachstum (gemessen in Fahrleistungen) herzustellen. Übergeordnetes Ziel der Verkehrspolitik sei die sustainability im Verkehrsbereich im Rahmen eines marktwirtschaftlichen Ansatzes. Gelinge dies nicht, so werde es eine neue Phase staatlichen Interventionismus geben. Eine gesamtwirtschaftliche Bewertung der Nutzen und Kosten des Verkehrs sei daher gerade im Hinblick auf das Ziel der sustainability und dessen marktwirtschaftliche Umsetzung zu leisten.

Interessenten für den Tagungsband wenden sich bitte an:

FGSV-Verlag GmbH, Köln, Fax: 0221-393747 